\title{
Mechanisms of Na adsorption on graphene and graphene oxide: density functional theory approach
}

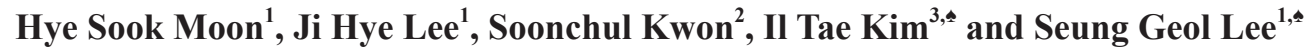 \\ ${ }^{1}$ Department of Organic Material Science and Engineering, Pusan National University, Busan 609-735, Korea \\ ${ }^{2}$ School of Civil and Environmental Engineering, Georgia Institute of Technology, Atlanta, GA 30332, USA \\ ${ }^{3}$ Department of Chemical and Biological Engineering, Gachon University, Seongnam 461-701, Korea
}

\section{Article Info}

Received 11 February 2015

Accepted 20 March 2015

*Corresponding Author

E-mail: itkim@gachon.ac.kr

E-mail: seunggeol.lee@pusan.ac.kr

Tel: $+82-31-750-8835$

Tel: $+82-51-510-2412$

\section{Open Access}

DOI: http://dx.doi.org/

10.5714/CL.2015.16.2.116

This is an Open Access article distributed under the terms of the Creative Commons Attribution Non-Commercial License (http://creativecommons.org/licenses/ by-nc/3.0/) which permits unrestricted non-commercial use, distribution, and reproduction in any medium, provided the original work is properly cited.

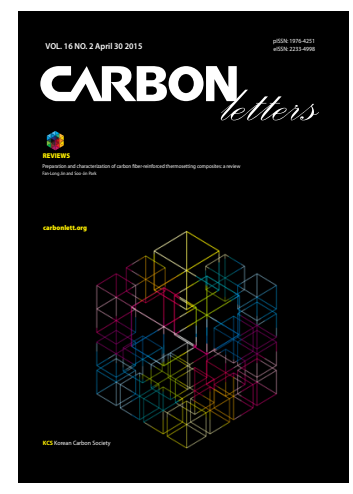

http://carbonlett.org

pISSN: $1976-4251$

elSSN: 2233-4998

Copyright $\odot$ Korean Carbon Society

\begin{abstract}
We investigated the adsorption of $\mathrm{Na}$ on graphene and graphene oxide, which are used as anode materials in sodium ion batteries, using density functional theory. The adsorption energy for $\mathrm{Na}$ on graphene was $-0.507 \mathrm{eV}$ at the hollow sites, implying that adsorption was favorable. In the case of graphene oxide, $\mathrm{Na}$ atoms were separately adsorbed on the epoxide and hydroxyl functional groups. The adsorption of $\mathrm{Na}$ on graphene oxide-epoxide (adsorption energy of $-1.024 \mathrm{eV}$ ) was found to be stronger than the adsorption of $\mathrm{Na}$ on pristine graphene. However, the adsorption of $\mathrm{Na}$ on graphene oxide-hydroxyl resulted in the generation of $\mathrm{NaOH}$ as a by-product. Using density of states (DOS) calculations, we found that the DOS of the Na-adsorbed graphene was shifted down more than that of the Na-adsorbed graphene oxide-epoxide. In addition, the intensity of the DOS around the Fermi level for the $\mathrm{Na}$-adsorbed graphene was higher than that for the Na-adsorbed graphene oxide-epoxide.
\end{abstract}

Key words: sodium ion battery, anode, graphene, graphene oxide, density functional theory

\section{Introduction}

Lithium ion batteries (LIBs) are essential energy storage systems present in a number of devices used in our daily lives such as laptops and mobile phones. However, the developments made in energy storage technology have outpaced the increase in energy storage needs [1]. Energy storage technologies such as Li batteries are considered as alternatives to fossil fuels, which are rapidly depleting all over the world. However, because $\mathrm{Li}$ is a scarce resource on Earth, producing large-sized LIBs is limited by the exponential increase in its cost [2]. In this context, sodium ion batteries (SIBs) are excellent alternatives to LIBs because of their lower cost and the greater availability of $\mathrm{Na}$ compared to Li. In fact, $\mathrm{Na}$ is abundantly available and the de-insertion behavior of $\mathrm{Na}$ in SIBs is very similar to that of Li in LIBs [3]. Hard carbon materials that have graphite layers and amorphous regions are widely used as anodes in SIBs [4]. In addition, carbon black, amorphous carbon, mesoporous carbon, and carbon fiber are being experimentally tested as anode materials for SIBs [5]. Among the various carbon materials, graphene, which has excellent electrical characteristics and physical properties, is gaining attention as an anode material candidate for SIBs. In a recent study, David et al. [6] synthesized layered freestanding papers comprised of acid-exfoliated few-layer molybdenum disulfide $\left(\mathrm{MoS}_{2}\right)$ and reduced graphene oxide (rGO) flakes for use as selfstanding flexible electrodes in SIBs. The electrode showed a good $\mathrm{Na}$ cycling ability with a stable charge capacity of approximately $230 \mathrm{mAh} \mathrm{g}^{-1}$ taking into account the total weight of the electrode and exhibited coulombic efficiencies up to approximately $99 \%$. In addition, static uniaxial tensile tests performed on the crumpled composite papers showed high average strains to failure up to approximately $2 \%$. Xie et al. [7] synthe- 
sized $\mathrm{SnS}_{2}$ nanoplatelets at graphene nanocomposites using a morphology controlled hydrothermal method. When $\mathrm{SnS}_{2} /$ graphene nanosheets were used as anode materials in SIBs, a high reversible specific Na-ion storage capacity of $725 \mathrm{mAh}$ $\mathrm{g}^{-1}$, stable cyclability, and an enhanced high-rate capability were observed.

Although such studies exist, the detailed mechanisms on $\mathrm{Na}$ adsorption on graphene and graphene oxide have not been fully elucidated yet. Therefore, in this work, we investigated the $\mathrm{Na}$ adsorption mechanisms on graphene-based anodes used in SIBs at the atomistic level by analyzing the adsorption energies and electronic properties using density functional theory (DFT).

\section{Experimental}

The DFT calculations were performed using the Vienna Ab-initio Simulation Package (VASP) [8,9] with the projector augmented wave (PAW) method [10], and generalized gradient approximation (GGA) and Perdew-Burke-Ernzerhof (PBE) functionals $[11,12]$. The PBE functional has been used to successfully describe the interactions between an adsorbate and various surfaces [13-15] including $\mathrm{sp}^{2}$ carbon-based materials [16-19].

The energy cut-off was set at $500 \mathrm{eV}$, and the cell size was $9.840 \times 9.840 \times 15 \AA$. The calculations were performed under the periodic boundary conditions, with the vacuum separation distance set to $15 \AA$ along the $\mathrm{z}$-direction, to avoid interactions between the images. In the energy and electronic property calculations, k-point samplings for the Brillouin zone were performed using the $5 \times 5 \times 1$ Monkhorst-Pack k-point mesh [20]. The atomic positions were optimized until the change in energy was less than $1 \times 10^{-6} \mathrm{eV}$ per cell, and the force on each atom was less than $0.01 \mathrm{eV} \AA^{-1}$. Spin-polarization and dipole corrections were also incorporated into the calculations. The adsorption energies of $\mathrm{Na}-$ adsorbed graphene and graphene oxide were defined by Eq. (1):

$$
E_{\text {ads }}=E_{\text {Graphene(oxide) }+\mathrm{Na}}-E_{\text {Graphene(oxide) }}-E_{\mathrm{Na}}
$$

, where $\mathrm{E}_{\text {Graphene(oxide)+Na }}$ is the total energy of the Na-adsorbed graphene or graphene oxide; $\mathrm{E}_{\text {Graphene(oxide) }}$ is the total energy of the graphene or graphene oxide, and $\mathrm{E}_{\mathrm{Na}}$ is the total energy of the isolated $\mathrm{Na}$. Dispersion corrections [21] were also considered in all the calculations. Charge transfer was calculated using the density derived electrostatic and chemical (DDEC) method $[22,23]$. Graphene oxide was assumed to consist of epoxide and hydroxyl oxidation functional groups, and each group was introduced to the pristine graphene shown in Fig. 1.

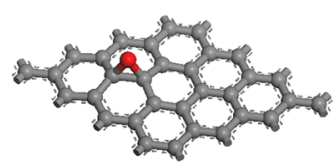

carbon

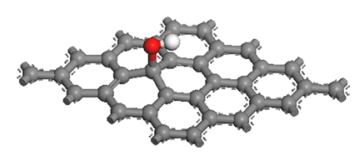

hydrogen
Fig. 1. Optimized structure of graphene oxide: (a) epoxide group and (b) hydroxyl group.

\section{Results and Discussion}

\subsection{Adsorption energies and charge analysis}

$\mathrm{Na}$ atoms were adsorbed onto hollow, bridge, and top sites of pristine graphene to determine the most stable position (Fig. 2). The hollow site was the most stable position for $\mathrm{Na}$ atoms, and the adsorption energy was $-0.507 \mathrm{eV}$. After $\mathrm{Na}$ adsorption, the optimized distance between the graphene and Na was $2.29 \AA$. The bridge sites were the second most stable adsorption sites for $\mathrm{Na}$ with an adsorption energy of -0.504 $\mathrm{eV}$, whereas adsorption onto the top sites was the least stable with an adsorption energy of $-0.493 \mathrm{eV}$. These results were confirmed by charge analysis showing that at all three sites, the charge was transferred from the $\mathrm{Na}$ to the graphene after adsorption, and the number of DDEC charges of the graphene at the hollow, bridge, and top sites was $-0.657 e,-0.515 e$, and $-0.508 e$, respectively. These results agreed well with the adsorption energy values. The adsorption energies, optimized distance between the $\mathrm{Na}$ atoms and the graphene, and charge distribution of the hollow, bridge, and top sites of the graphene are summarized in Fig. $3 \mathrm{a}$ and Table 1.

Graphene oxide was assumed to contain epoxide and hydroxyl functional groups, and $\mathrm{Na}$ was adsorbed onto each of these types of functional groups on the graphene oxide. First, $\mathrm{Na}$ was adsorbed vertically onto the oxygen atoms of the graphene oxide-epoxide (GO-epoxide). The adsorption energy for this case was $-1.024 \mathrm{eV}$, which was approximately twice
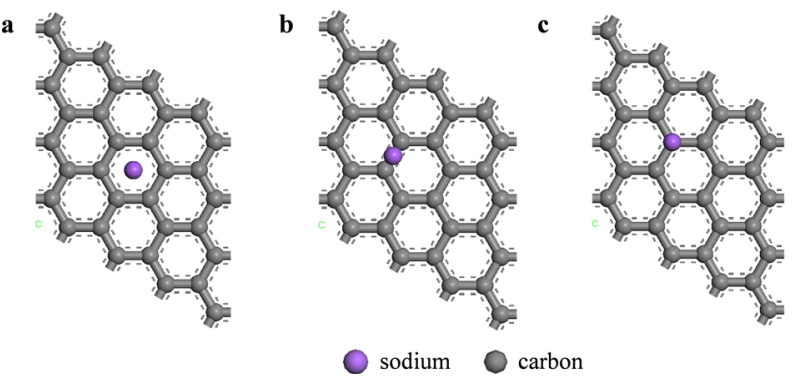

Fig. 2. Na adsorption sites on the graphene: (a) hollow, (b) bridge, and (c) top.
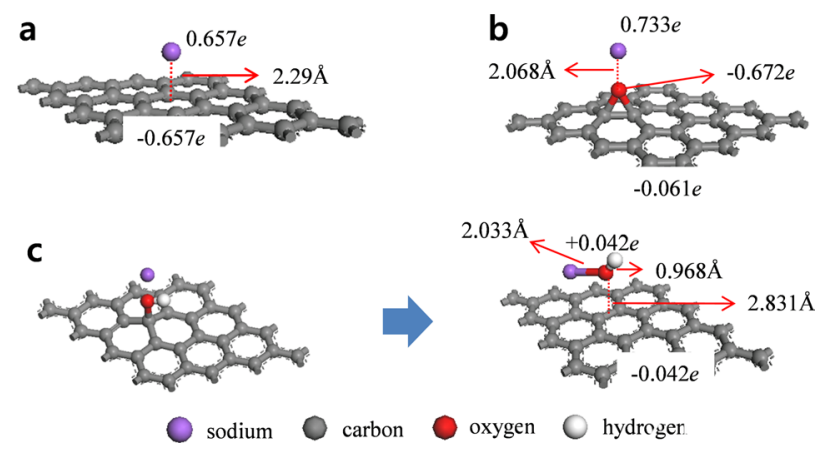

Fig. 3. The optimized structures of the Na-adsorbed graphene and graphene oxide: (a) $\mathrm{Na}$ adsorption on graphene, (b) $\mathrm{Na}$ adsorption on graphene oxide-epoxide, and (c) initial and final structures of the Naadsorbed graphene oxide-hydroxyl before and after $\mathrm{Na}$ adsorption. 
Table 1. Adsorption energy, distance between $\mathrm{Na}$ atoms and graphene, and charge distribution of the bridge, hollow, and top sites on the graphene

\begin{tabular}{ccccc} 
Site & $\begin{array}{c}\text { Adsorption } \\
\text { energy }(\mathrm{eV})\end{array}$ & Distance $(\AA)$ & \multicolumn{2}{c}{ Charge transfer $(e)$} \\
\cline { 4 - 5 } Bridge & -0.504 & 2.548 & 0.515 & -0.515 \\
Hollow & -0.507 & 2.285 & 0.657 & -0.657 \\
Top & -0.493 & 2.563 & 0.508 & -0.508 \\
\hline
\end{tabular}

Table 2. Charge distribution on the Na-adsorbed graphene oxide (epoxide and hydroxyl) compared with those on the pristine graphene oxide (epoxide and hydroxyl) and $\mathrm{NaOH}$

\begin{tabular}{cccccc} 
& \multicolumn{5}{c}{ Charges $(e)$} \\
\hline \multirow{2}{*}{ Atoms } & \multicolumn{2}{c}{ GO-epoxide } & \multicolumn{3}{c}{ GO-hydroxyl } \\
\cline { 2 - 6 } & Initial & $\begin{array}{c}\text { After Na } \\
\text { adsorption }\end{array}$ & Initial & $\begin{array}{c}\text { After Na } \\
\text { adsorption }\end{array}$ & $\begin{array}{c}\mathrm{NaOH} \\
\text { molecule }\end{array}$ \\
\hline $\begin{array}{c}\mathrm{C} \\
\text { (graphene) }\end{array}$ & 0.287 & -0.061 & 0.201 & -0.042 & - \\
$\mathrm{Na}$ & - & 0.733 & - & 0.912 & 0.922 \\
$\mathrm{O}$ & -0.287 & -0.672 & -0.569 & -1.245 & -1.308 \\
$\mathrm{H}$ & - & - & 0.368 & 0.375 & 0.385 \\
\hline
\end{tabular}

GO: graphene oxide.

the value for the adsorption of $\mathrm{Na}$ onto the pristine graphene. We believe that the oxygen in the epoxide caused a doping effect, and subsequently, the adsorption energy was enhanced In addition, the optimized distance between the graphene and $\mathrm{Na}$ was $2.068 \AA$. The optimized structure of the Na-adsorbed GO-epoxide and the charge transfer values for the graphene oxide and Na-adsorbed graphene oxide are summarized in Fig. $3 \mathrm{~b}$ and Table 2 . The sum of the charges of the oxygen and carbon in the GO-epoxide was $-0.287 e$ and $+0.287 e$ before $\mathrm{Na}$ adsorption, respectively. However, after $\mathrm{Na}$ adsorption, the sum of the charges of the oxygen and carbon in the GO-epoxide was $-0.672 e$ and $-0.061 e$, respectively. In other words, the oxygen and carbon in the GO-epoxide gained charges of $-0.385 e$ and $-0.348 e$, respectively, as a result of the $\mathrm{Na}$ adsorption. Thus, we found that a total charge of $+0.733 e$ was transferred from the $\mathrm{Na}$ to the oxygen and carbon in the graphene oxide during adsorption.

$\mathrm{Next}, \mathrm{Na}$ was adsorbed vertically onto the oxygen atom of the hydroxyl group in the graphene oxide-hydroxyl (GO-hydroxyl). Unlike graphene and GO-epoxide, the hydroxyl group dissociated as a result of $\mathrm{Na}$ adsorption and produced $\mathrm{NaOH}$ as a byproduct (Fig. 3c). The optimized distance between the oxygen of the $\mathrm{NaOH}$ and graphene was $2.831 \AA$, and the adsorption energy between the $\mathrm{NaOH}$ and graphene was $-0.402 \mathrm{eV}$. Kim et al. [24] showed that the epoxide groups are much more stable than the hydroxyl groups in graphene oxide by DFT with the GGA-PW91 functional. Because of the low binding energy, a hydroxyl group in the interior aromatic domain of the graphene oxide is unstable, and as a consequence, the hydroxyl group can be dissociated $[25,26]$. However, it should be noted that because the present investigation is based on DFT calculations that focus on the ground state of Na-graphene systems, the results obtained during the operation of SIBs may be different. Dissociated $\mathrm{NaOH}$ has a similar charge distribution as an isolated $\mathrm{NaOH}$ molecule. As a result, charges of $-0.042 e$ were transferred from the $\mathrm{NaOH}$ to the graphene. The small amount of charge transferred implies that the $\mathrm{NaOH}$ and graphene molecules interacted via weak physical adsorption.

\subsection{Electronic properties}

We investigated the electronic properties of the graphene and graphene oxide by analyzing the density of states (DOS). The Fermi levels for all the DOS shifted to zero. The DOS of pristine and Na-adsorbed graphene are shown in Fig. 4a. After $\mathrm{Na}$ adsorption, however, the DOS of the Na-adsorbed graphene shifted down, and their intensity near the Fermi level increased, indicating that more electronic states can exist at the Fermi level. This could be attributed to the fact that electrons from $\mathrm{Na}$ are injected towards the graphene.

Fig. $4 \mathrm{~b}$ shows the DOS of the GO-epoxide and Na-adsorbed GO-epoxide. The Dirac point was not observed in the DOS of the GO-epoxide because the $2 p$ orbital of the oxygen hybridized with the $\pi$-orbital of the graphene, leading to a change in the Dirac point [27]. After Na adsorption, all the DOS were shifted down, and the intensity of DOS near the Fermi level increased, similar to the case of Na-adsorbed graphene. These results were also attributed to the injection of electrons from the $\mathrm{Na}$ to the GO-epoxide.

Fig. 4c shows the DOS of the pristine GO-hydroxyl and Naadsorbed GO-hydroxyl. While the DOS of the GO-hydroxyl exhibited several peaks between -2 and $2 \mathrm{eV}$, the DOS of the Naadsorbed GO-hydroxyl exhibited a broad line between -2 and 2 $\mathrm{eV}$, similar to the case of the graphene. In addition, the intensity of the DOS near the Fermi level was significantly increased after $\mathrm{Na}$ adsorption. To investigate this in more detail, we calculated the local density of states (LDOS) of the carbon (graphene) after $\mathrm{Na}$ adsorption and the $\mathrm{NaOH}$ molecules (Fig. 4d). The LDOS of the carbon, shown in Fig. 4d, was similar to that of the graphene. Therefore, we concluded that the GO-hydroxyl reverted to the graphene, as a result of the dissociation of $\mathrm{OH}$. In addition, a broad line between -2 and $2 \mathrm{eV}$ in the DOS of the Na-adsorbed GO-hydroxyl was a result of the LDOS of the carbon. In the case of the LDOS of the $\mathrm{NaOH}$ molecules, the DOS of the $\mathrm{NaOH}$ exhibited a huge peak at the Fermi level, providing the energy levels of the molecular orbitals for the $\mathrm{NaOH}$ molecules rather than the band structure. Therefore, in Fig. 4c, the intensity of the DOS peak around the Fermi level was significantly increased after $\mathrm{Na}$ adsorption, which appears to be affected by the DOS of the dissociated $\mathrm{NaOH}$.

As a result, the DOS of the Na-adsorbed graphene shifted down more than that of the GO-epoxide, and the intensity of the DOS around the Fermi level was higher than that of the Naadsorbed GO-epoxide. Although the adsorption energy for $\mathrm{Na}$ onto GO-epoxide was higher than the corresponding value for graphene, after $\mathrm{Na}$ adsorption, the electronic properties of the graphene were better than those of the GO-epoxide because oxygen doping weakens the $\mathrm{sp}^{2}$ - hybridization of the carbon atoms 

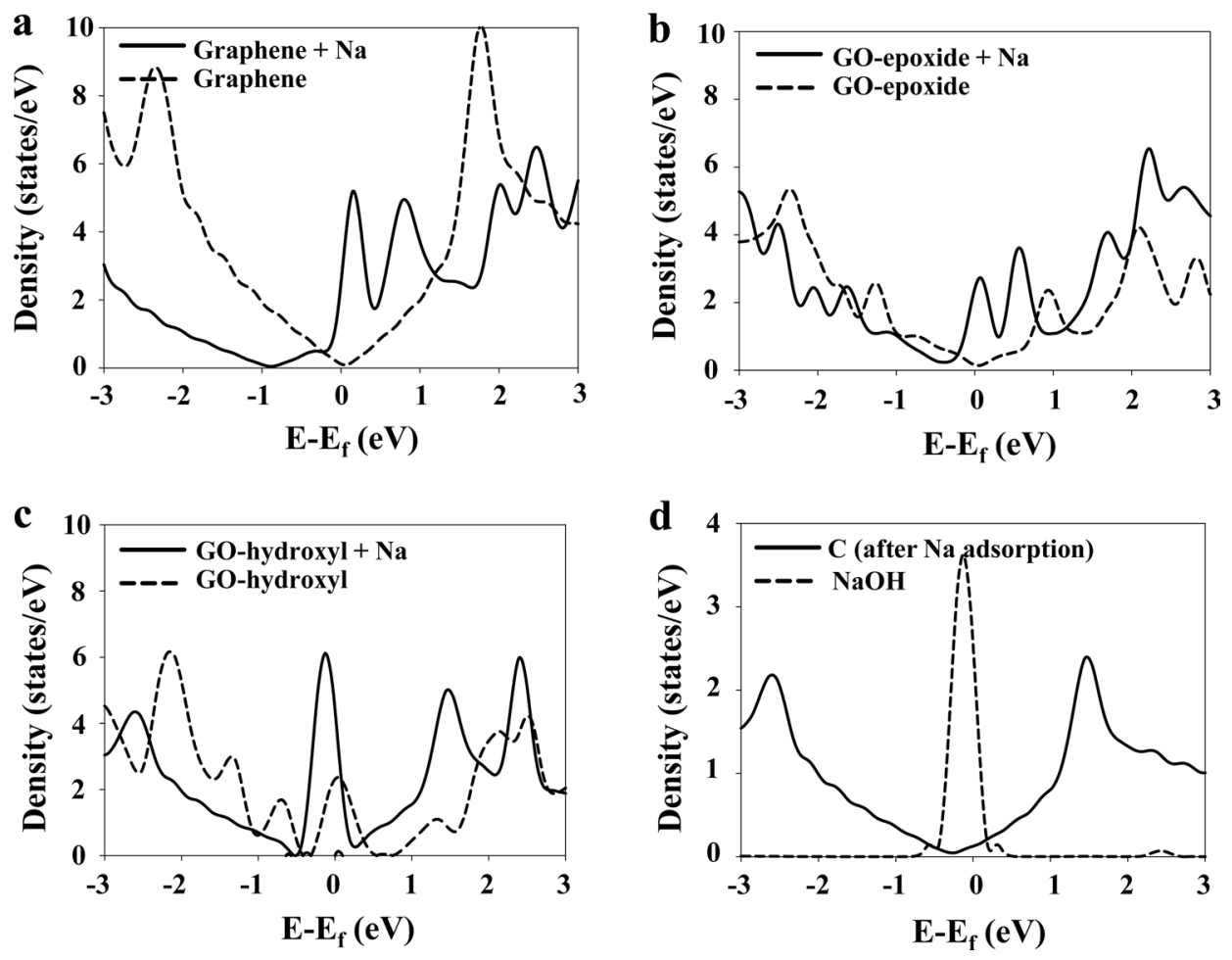

Fig. 4. The density of states (DOS) of the graphene and graphene oxide (GO) systems: (a) graphene and Na-adsorbed graphene, (b) GO-epoxide and Naadsorbed GO-epoxide, (c) GO-hydroxyl and Na-adsorbed GO-hydroxyl, and (d) local DOS of the NaOH molecules and the carbon of the Na-adsorbed GOhydroxyl.

in the GO. In the case of the GO-hydroxyl, after Na adsorption, the $\mathrm{OH}$ group dissociated and produced $\mathrm{NaOH}$ molecules. In addition, the dissociated $\mathrm{NaOH}$ molecules were adsorbed onto the graphene by weak physical adsorption.

\section{Conclusions}

In this paper, we investigated the adsorption energies and electronic properties for the adsorption of $\mathrm{Na}$ onto graphene and graphene oxide, using the first-principles DFT method. $\mathrm{Na}$ adsorption was the most stable at the hollow sites of pristine graphene with an adsorption energy of $-0.507 \mathrm{eV}$. In the case of the GO-epoxide, the $\mathrm{Na}$ adsorption energy was -1.024 $\mathrm{eV}$, which was more negative than the adsorption energy for $\mathrm{Na}$ onto pristine graphene. In the case of the GO-hydroxyl, the hydroxyl group dissociated from the GO-hydroxyl, and $\mathrm{NaOH}$ was formed as a by-product. After $\mathrm{Na}$ adsorption, the DOS of both graphene and GO-epoxide shifted down, and the intensity of the DOS around the Fermi level increased due to electron injection in both cases. In addition, it was found that the DOS of the Na-adsorbed graphene shifted down more than that of the GO-epoxide, and the intensity of the DOS around the Fermi level for the Na-adsorbed graphene was higher than that for the Na-adsorbed GO-epoxide. Therefore, after $\mathrm{Na}$ adsorption, the electronic properties of the graphene were better than those of the GO-epoxide because of oxygen doping which weakened the $\mathrm{sp}^{2}$-hybridization of the carbon atoms in the GO.

\section{Acknowledgements}

This research was supported by the Basic Science Research Program through the National Research Foundation of Korea (NRF) funded by the Ministry of Science, ICT \& Future Planning (2014R1A1A1004096). This research was supported by the Gachon University research fund of 2014 (GCU-2014-0122).

\section{References}

[1] Slater MD, Kim D, Lee E, Johnson CS. Sodium-ion batteries. Adv Funct Mater, 23, 947 (2013). http://dx.doi.org/10.1002/ adfm. 201200691.

[2] Ellis BL, Nazar LF. Sodium and sodium-ion energy storage batteries. Curr Opin Solid State Mater Sci, 16, 168 (2012). http://dx.doi. org/10.1016/j.cossms.2012.04.002.

[3] Palomares V, Casas-Cabanas M, Castillo-Martinez E, Han MH, Rojo T. Update on Na-based battery materials. A growing research path. Energy Environ Sci, 6, 2312 (2013). http://dx.doi. org/10.1039/C3ee41031e.

[4] Kim SW, Seo DH, Ma XH, Ceder G, Kang K. Electrode materials for rechargeable sodium-ion batteries: potential alternatives to current lithium-ion batteries. Adv Energy Mater, 2, 710 (2012). http:// dx.doi.org/10.1002/aenm.201200026.

[5] Palomares V, Serras P, Villaluenga I, Hueso KB, Carretero-Gonzalez J, Rojo T. Na-ion batteries, recent advances and present challenges to become low cost energy storage systems. Energy Environ 
Sci, 5, 5884 (2012). http://dx.doi.org/10.1039/C2ee02781j.

[6] David L, Bhandavat R, Singh G. $\mathrm{MoS}_{2}$ /graphene composite paper for sodium-ion battery electrodes. Acs Nano, 8, 1759 (2014). http://dx.doi.org/10.1021/Nn406156b.

[7] Xie XQ, Su DW, Chen SQ, Zhang JQ, Dou SX, Wang GX. $\mathrm{SnS}_{2}$ nanoplatelet@graphene nanocomposites as high-capacity anode materials for sodium-ion batteries. Chem Asian J, 9, 1611 (2014). http://dx.doi.org/10.1002/asia.201400018.

[8] Kresse G, Furthmuller J. Efficient iterative schemes for ab initio total-energy calculations using a plane-wave basis set. Phys Rev B, 54, 11169 (1996). http://dx.doi.org/10.1103/PhysRevB.54.11169.

[9] Kresse G, Hafner J. Abinitio molecular-dynamics for liquid-metals. Phys Rev B, 47, 558 (1993). http://dx.doi.org/10.1103/PhysRevB.47.558.

[10] Kresse G, Joubert D. From ultrasoft pseudopotentials to the projector augmented-wave method. Phys Rev B, 59, 1758 (1999). http:/ dx.doi.org/10.1103/PhysRevB.59.1758.

[11] Perdew JP, Burke K, Ernzerhof M. Generalized gradient approximation made simple. Phys Rev Lett, 77, 3865 (1996). http://dx.doi. org/10.1103/PhysRevLett.77.3865.

[12] Perdew JP, Burke K, Wang Y. Generalized gradient approximation for the exchange-correlation hole of a many-electron system. Phys Rev B, 54, 16533 (1996). http://dx.doi.org/10.1103/PhysRevB.54.16533.

[13] Kwon S, Choi JI, Lee SG, Jang SS. A density functional theory (DFT) study of $\mathrm{CO}_{2}$ adsorption on Mg-rich minerals by enhanced charge distribution. Comput Mater Sci, 95, 181 (2014). http:// dx.doi.org/10.1016/j.commatsci.2014.07.042.

[14] Lee SG, Choi JI, Koh W, Jang SS. Adsorption of beta-D-glucose and cellobiose on kaolinite surfaces: density functional theory (DFT) approach. Appl Clay Sci, 71, 73 (2013). http://dx.doi. org/10.1016/j.clay.2012.11.002.

[15] Kwon S, Lee SG, Chung E, Lee WR. $\mathrm{CO}_{2}$ adsorption on $\mathrm{H}_{2} \mathrm{O}$-saturated $\mathrm{BaO}\left(\begin{array}{lll}1 & 0 & 0\end{array}\right)$ and induced barium surface dissociation. Bull Korean Chem Soc, 36, 11 (2015).

[16] Koh W, Choi JI, Donaher K, Lee SG, Jang SS. Mechanism of Li adsorption on carbon nanotube-fullerene hybrid system: a firstprinciples study. ACS Appl Mater Interfaces, 3, 1186 (2011). http:// dx.doi.org/10.1021/Am200018w.

[17] Koh W, Choi JI, Jeong E, Lee SG, Jang SS. Li adsorption on a
Fullerene-Single wall carbon nanotube hybrid system: density functional theory approach. Curr Appl Phys, 14, 1748 (2014). http://dx.doi.org/10.1016/j.cap.2014.09.031.

[18] Koh W, Moon HS, Lee SG, Choi JI, Jang SS. A first-principles study of lithium adsorption on a graphene-fullerene nanohybrid system. ChemPhysChem, 16, 789 (2015). http://dx.doi. org/10.1002/cphc.201402675.

[19] Koh W, Choi JI, Lee SG, Lee WR, Jang SS. First-principles study of $\mathrm{Li}$ adsorption in a carbon nanotube-fullerene hybrid system. Carbon, 49, 286 (2011). http://dx.doi.org/10.1016/j.carbon.2010.09.022.

[20] Monkhorst HJ, Pack JD. Special points for brillouin-zone integrations. Phys Rev B, 13, 5188 (1976). http://dx.doi.org/10.1103/ PhysRevB.13.5188.

[21] Grimme S, Antony J, Ehrlich S, Krieg H. A consistent and accurate $a b$ initio parametrization of density functional dispersion correction (DFT-D) for the 94 elements H-Pu. J Chem Phys, 132, 154104 (2010). http://dx.doi.org/10.1063/1.3382344.

[22] Manz TA, Sholl DS. Improved atoms-in-molecule charge partitioning functional for simultaneously reproducing the electrostatic potential and chemical states in periodic and nonperiodic materials. J Chem Theory Comput, 8, 2844 (2012). http://dx.doi.org/10.1021/ Ct3002199.

[23] Manz TA, Sholl DS. Chemically meaningful atomic charges that reproduce the electrostatic potential in periodic and nonperiodic materials. J Chem Theory Comput, 6, 2455 (2010). http://dx.doi. org $/ 10.1021 / \mathrm{Ct} 100125 \mathrm{x}$

[24] Kim MC, Hwang GS, Ruoff RS. Epoxide reduction with hydrazine on graphene: a first principles study. J Chem Phys, 131, 064704 (2009). http://dx.doi.org/10.1063/1.3197007.

[25] Pei SF, Cheng HM. The reduction of graphene oxide. Carbon, 50, 3210 (2012). http://dx.doi.org/10.1016/j.carbon.2011.11.010.

[26] Gao XF, Jang J, Nagase S. Hydrazine and thermal reduction of graphene oxide: reaction mechanisms, product structures, and reaction design. J Phys Chem C, 114, 832 (2010). http://dx.doi.org/10.1021/ Jp909284g.

[27] Yan JA, Chou MY. Oxidation functional groups on graphene: structural and electronic properties. Phys Rev B, 82, 125403 (2010) http://dx.doi.org/10.1103/Physrevb.82.125403. 